Bull. Korean Math. Soc. 45 (2008), No. 4, pp. 615-620

\title{
NEW CHARACTERIZATION OF THE CLASS OF STARLIKE FUNCTIONS
}

\author{
Emel Yavuz, Yaşar Polatoũlu, and Shigeyoshi Owa
}

Abstract. For analytic functions $f(z)$ in the open unit disc $\mathbb{D}$, an operator $\mathcal{N}_{\alpha}(f(z))$ relating with starlike functions is introduced. The object of the present paper is to discuss some properties of the operator $\mathcal{N}_{\alpha}(f(z))$.

\section{Introduction}

Let $\Omega$ be the class of functions $w(z)$ which are regular in the open unit disc $\mathbb{D}=\{z \in \mathbb{C}|| z \mid<1\}$ and satisfy $w(0)=0$ and $|w(z)|<1$ for all $z \in \mathbb{D}$.

For $w(z) \in \Omega$, let $\mathcal{P}$ denote the family of functions $p(z)$ of the form

$$
p(z)=1+\sum_{n=1}^{\infty} p_{n} z^{n}
$$

that are regular in $\mathbb{D}$ satisfying

$$
p(z)=\frac{1+w(z)}{1-w(z)} \quad(z \in \mathbb{D}) .
$$

A function $p(z) \in \mathcal{P}$ is called as Carathéodory function in $\mathbb{D}(c f$. [1], [2]).

Further, let $\mathcal{A}$ be the class of functions $f(z)$ of the form

$$
f(z)=z+\sum_{n=2}^{\infty} a_{n} z^{n}
$$

which are analytic in $\mathbb{D}$. A function $f(z) \in \mathcal{A}$ is said to be starlike of order $\alpha$ in $\mathbb{D}$ if it satisfies

$$
\operatorname{Re}\left\{\frac{z f^{\prime}(z)}{f(z)}\right\}>\alpha \quad(z \in \mathbb{D})
$$

for some $\alpha(0 \leqq \alpha<1)$. We denote by $\mathcal{S}^{*}(\alpha)$ the subclass of $\mathcal{A}$ consisting of all starlike functions of order $\alpha$ in $\mathbb{D}$.

For $h(z)$ and $s(z)$ in the class $\mathcal{A}, h(z)$ is said to be subordinate to $s(z)$, written by $h(z) \prec s(z)$, if there exists $w(z) \in \Omega$ such that $h(z)=s(w(z))$

Received March 7, 2007; Revised October 15, 2007.

2000 Mathematics Subject Classification. Primary 30C45.

Key words and phrases. Carathéodory function, starlike function, operator. 
for $z \in \mathbb{D}$. In particular, if $s(z)$ is univalent in $\mathbb{D}$, then the subordination $h(z) \prec s(z)$ is equivalent to $h(0)=s(0)$ and $h(\mathbb{D}) \subset s(\mathbb{D})$.

For $f(z) \in \mathcal{A}$, let us introduce the operator $\mathcal{N}_{\alpha}(f(z))$ defined by

$$
\mathcal{N}_{\alpha}(f(z))=z-2 \alpha \frac{f(z)}{f^{\prime}(z)} \quad(z \in \mathbb{D})
$$

for some $\alpha(0 \leqq \alpha<1)$. If $\alpha=\frac{1}{2}$, the operator

$$
\mathcal{N}_{\frac{1}{2}}(f(z))=z-\frac{f(z)}{f^{\prime}(z)} \quad(z \in \mathbb{D})
$$

is said to be Newtonian.

\section{Properties of $\mathcal{N}_{\alpha}(f(z))$}

To discuss some properties of the operator $\mathcal{N}_{\alpha}(f(z))$, we have to recall here the following lemma for Carathéodory functions (see [3]).

Lemma 2.1. If $p(z) \in \mathcal{P}$, then

$$
\frac{1-|z|}{1+|z|} \leqq|p(z)| \leqq \frac{1+|z|}{1-|z|}
$$

and

$$
\left|p^{\prime}(z)\right| \leqq \frac{2}{(1-|z|)^{2}}
$$

for $z \in \mathbb{D}$.

Now we derive

Theorem 2.2. If $f(z) \in \mathcal{A}$ satisfies

$$
\left|\left(\frac{\mathcal{N}_{\alpha}(f(z))}{z}\right)^{\prime}\right| \leqq \beta|z|^{\gamma} \quad(z \in \mathbb{D})
$$

for some $\beta$ and $\gamma$ such that $0<\beta \leqq \gamma+1$ and $\gamma \geqq 0$, then $f(z) \in \mathcal{S}^{*}(\alpha)$.

Proof. For $f(z) \in \mathcal{A}$, we define the function $w(z)$ by

$$
w(z)=z\left(1-2 \alpha \frac{f(z)}{z f^{\prime}(z)}\right)=\mathcal{N}_{\alpha}(f(z)) \quad(z \in \mathbb{D}) .
$$

Then $w(z)$ is regular in $\mathbb{D}$ and $w(0)=0$.

Since $\mathcal{N}_{\alpha}(f(z))=w(z)$, the condition of the theorem gives us that

$$
\left|\left(\frac{\mathcal{N}_{\alpha}(f(z))}{z}\right)^{\prime}\right|=\left|\left(\frac{w(z)}{z}\right)^{\prime}\right| \leqq \beta|z|^{\gamma} \quad(z \in \mathbb{D}) .
$$


It follows from the above that

$$
\begin{aligned}
\left|\frac{w(z)}{z}\right| & =\left|\int_{0}^{z}\left(\frac{w(t)}{t}\right)^{\prime} d t\right| \\
& \leqq \int_{0}^{|z|} \beta|t|^{\gamma} d|t|=\frac{\beta}{\gamma+1}|z|^{\gamma+1} .
\end{aligned}
$$

This implies that

$$
\left|\frac{w(z)}{z}\right| \leqq \frac{\beta}{\gamma+1}|z|^{\gamma+1}<1 \quad(z \in \mathbb{D})
$$

because $0<\beta \leqq \gamma+1$ and $\gamma \geqq 0$. Therefore, by the definition for $w(z)$, we conclude that

which is equivalent to

$$
\left|2 \alpha \frac{f(z)}{z f^{\prime}(z)}-1\right|<1 \quad(z \in \mathbb{D}),
$$

$$
\operatorname{Re}\left(\frac{z f^{\prime}(z)}{f(z)}\right)>\alpha \quad(z \in \mathbb{D})
$$

Next, we show

Theorem 2.3. If $f(z) \in \mathcal{S}^{*}\left(\frac{1}{2}\right)$, then

$$
\left|\mathcal{N}_{\frac{1}{2}}(f(z))-\frac{1+|z|^{2}}{2}\right| \geqq \frac{1-|z|^{2}}{2} \quad(z \in \mathbb{D})
$$

and

$$
\left|\mathcal{N}_{\frac{1}{2}}(f(z))+\frac{1+|z|^{2}}{2}\right| \geqq \frac{1-|z|^{2}}{2} \quad(z \in \mathbb{D}) .
$$

Furthermore,

$$
\left|\left(\mathcal{N}_{\frac{1}{2}}(f(z))\right)^{\prime}\right| \leqq \frac{2|z|\left(1+|z|^{2}\right)}{(1-|z|)^{2}} \quad(z \in \mathbb{D}) .
$$

Proof. For $f(z) \in \mathcal{S}^{*}\left(\frac{1}{2}\right)$ let us define the function $w(z)$ by

$$
w(z)=1-\frac{f(z)}{z f^{\prime}(z)} \quad(z \in \mathbb{D})
$$

Then we see that $w(0)=0$ and

$$
\operatorname{Re}\left(\frac{z f^{\prime}(z)}{f(z)}\right)=\operatorname{Re}\left(\frac{1}{1-w(z)}\right)>\frac{1}{2} \quad(z \in \mathbb{D})
$$

This implies that $|w(z)|<1(z \in \mathbb{D})$ so that $w(z) \in \Omega$. If we define the function $p(z)$ by

$$
p(z)=\frac{1+w(z)}{1-w(z)},
$$


then $p(z) \in \mathcal{P}$. Applying Lemma 2.1 for $p(z)$, we have that

$$
\frac{1-|z|}{1+|z|} \leqq\left|\frac{1+w(z)}{1-w(z)}\right| \leqq \frac{1+|z|}{1-|z|} \quad(z \in \mathbb{D}) .
$$

From the right hand inequality, we see that

$$
\left|\mathcal{N}_{\frac{1}{2}}(f(z))-\frac{1+|z|^{2}}{2}\right| \geqq \frac{1-|z|^{2}}{2} \quad(z \in \mathbb{D}) .
$$

Also, from the left hand inequality, we have that

$$
\left|\mathcal{N}_{\frac{1}{2}}(f(z))+\frac{1+|z|^{2}}{2}\right| \geqq \frac{1-|z|^{2}}{2} \quad(z \in \mathbb{D}) .
$$

Further, Lemma 2.1 implies that

$$
\left|p^{\prime}(z)\right|=\left|\frac{2 w^{\prime}(z)}{(1-w(z))^{2}}\right| \leqq \frac{2}{(1-|z|)^{2}} \quad(z \in \mathbb{D}) .
$$

Since $|w(z)| \leqq|z|<1(z \in \mathbb{D})$, we obtain that

$$
\left|w^{\prime}(z)\right| \leqq\left(\frac{1+|z|}{1-|z|}\right)^{2} \quad(z \in \mathbb{D}) .
$$

Thus we see that

$$
\begin{aligned}
\left|\left(\mathcal{N}_{\frac{1}{2}}(f(z))\right)^{\prime}\right| & \leqq|w(z)|+\left|z w^{\prime}(z)\right| \\
& \leqq|z|\left(1+\left|w^{\prime}(z)\right|\right) \\
& \leqq \frac{2|z|\left(1+|z|^{2}\right)}{(1-|z|)^{2}}
\end{aligned}
$$

which completes the proof of the theorem.

To discuss our next problem, we need the following lemma in [2].

Lemma 2.4. If $w(z) \in \Omega$, then

$$
\left|w^{\prime}(z)\right| \leqq \begin{cases}1 & (0 \leqq|z| \leqq \sqrt{2}-1) \\ \frac{\left(1+|z|^{2}\right)^{2}}{4|z|(1-|z|)^{2}} & (\sqrt{2}-1 \leqq|z|<1)\end{cases}
$$

Next our result is contained in

Theorem 2.5. A function $f(z) \in \mathcal{A}$ is in the class $\mathcal{S}^{*}\left(\frac{1}{2}\right)$ for $0 \leqq|z| \leqq \sqrt{2}-1$ if and only if

$$
\left|\left(\mathcal{N}_{\frac{1}{2}}(f(z))\right)^{\prime}\right| \leqq 2|z| \quad(0 \leqq|z| \leqq \sqrt{2}-1) .
$$

Proof. Let us put $\alpha=\frac{1}{2}, \beta=2$ and $\gamma=1$ in Theorem 2.2. Then we see that $\left|\left(\mathcal{N}_{\frac{1}{2}}(f(z))\right)^{\prime}\right| \leqq 2|z|$ implies $f(z) \in \mathcal{S}^{*}\left(\frac{1}{2}\right)$. Furthermore, from the proof of Theorem 2.3, we have that $f(z) \in \mathcal{S}^{*}\left(\frac{1}{2}\right)$ implies

$$
\left|\left(\mathcal{N}_{\frac{1}{2}}(f(z))\right)^{\prime}\right| \leqq|z|\left(1+\left|w^{\prime}(z)\right|\right) \leqq 2|z|
$$


for $0 \leqq|z| \leqq \sqrt{2}-1$.

Finally, in view of Theorem 2.5, we derive the following corollaries.

Corollary 2.6. If $f \in \mathcal{S}^{*}\left(\frac{1}{2}\right)$ for $0 \leqq|z| \leqq \sqrt{2}-1$, then

$$
\left|\frac{f^{\prime \prime}(z)}{f^{\prime}(z)}\right| \leqq 2\left|\frac{z f^{\prime}(z)}{f(z)}\right| \quad(0 \leqq|z| \leqq \sqrt{2}-1) .
$$

Proof. Note that

$$
\mathcal{N}_{\frac{1}{2}}(f(z))=z-\frac{f(z)}{f^{\prime}(z)} \quad(z \in \mathbb{D}) .
$$

Therefore, Theorem 2.5 shows that

$$
\left|\left(\mathcal{N}_{\frac{1}{2}}(f(z))\right)^{\prime}\right|=\left|\frac{\frac{f^{\prime \prime}(z)}{f^{\prime}(z)}}{\frac{f^{\prime}(z)}{f(z)}}\right| \leqq 2|z|,
$$

that is, that

$$
\left|\frac{f^{\prime \prime}(z)}{f^{\prime}(z)}\right| \leqq 2\left|\frac{z f^{\prime}(z)}{f(z)}\right|(0 \leqq|z| \leqq \sqrt{2}-1)
$$

Corollary 2.7. If $f(z) \in \mathcal{S}^{*}\left(\frac{1}{2}\right)$ for $0 \leqq|z| \leqq \sqrt{2}-1$, then

$$
\left|\frac{f^{\prime \prime}(z)}{f^{\prime}(z)}\right|<\frac{2}{1-|z|} \quad(0 \leqq|z| \leqq \sqrt{2}-1) .
$$

Proof. Since $f(z) \in \mathcal{S}^{*}\left(\frac{1}{2}\right)$ implies that

$$
\frac{z f^{\prime}(z)}{f(z)} \prec \frac{1}{1-z} \quad(0 \leqq|z| \leqq \sqrt{2}-1),
$$

we obtain that

$$
\left|\frac{z f^{\prime}(z)}{f(z)}-\frac{1}{1-|z|^{2}}\right|<\frac{|z|}{1-|z|^{2}} \quad(0 \leqq|z| \leqq \sqrt{2}-1) .
$$

Therefore, applying Corollary 2.6, we complete the proof of the corollary.

Remark 2.8. We note that the convex function is starlike of order $\frac{1}{2}$. Therefore, all convex functions $f(z) \in \mathcal{A}$ satisfy Corollary 2.6 and Corollary 2.7 for $0 \leqq$ $|z| \leqq \sqrt{2}-1$.

\section{References}

[1] C. Carathéodory, Überden Variabilitätsbereich der Fourier'schen Konstanten von Positiven Harmonischen Funktionen, Rend. Civc. Mat. Palermo, 32 (1911), 193-217.

[2] P. L. Duren, Univalent Functions, Grundlehren der Mathematischen Wissenschaften 259, Springer-Verlag, New York, 1983.

[3] A. W. Goodman, Univalent Functions, Vol. I, Mariner Publishing Co., Inc., Tampa, FL, 1983. 
Emel Yavuz

Department of Mathematics and Computer Science

TC İstanbul KÜLtür University

34156 İstanbul, Turkey

E-mail address: e.yavuz@iku.edu.tr

YaŞar Polato $\tilde{L}$ LU

Department of Mathematics and Computer Science

TC İstanbul KÜltür University

34156 İstanbul, TURKEY

E-mail address: y.polatoglu@iku.edu.tr

Shigeyoshi OWA

Department of Mathematics

KINKI UNIVERSITY

Higashi-Osaka, Osaka 577-8502, JAPAN

E-mail address: owa@math.kindai.ac.jp 